\title{
New journal for great advances in Eye Care
}

\author{
Manuel G. Penedo ${ }^{1 *}$ \\ ${ }^{1}$ Director of the CITIC Research Center, University of Coruña, Spain
}

The most recent estimate by WHO about the global dimension and the causes for visual disability confirm that there is an excellent opportunity to change the life of millions of people: an $80 \%$ of the causes for visual disability are preventable and curable. The WHO calculates that in 2010, 285 million people suffer from visual disability, of which 39 million were blind. In the last years, there has been a lot progress in many fields thanks to research and innovation developed in hospitals, Universities, Research centres, Private Entities, etc... which have meant an improvement in the visual care of our society. An example of the mentioned progress are the improvements of the medical treatments and the advances of the therapeutic arsenal in retinal disease, like the antiangiogenic drugs or the use of gradual corticosteroides release devices. In the surgical field, very important progress has been made as for example, in the myopia treatment (myopic foveoschisis), where the high blindness occurrence is worrying, in cornea, where a better visual acuity is being looked for and the irregular cornea through operations with laser treatment guided by corneal topography, or in cataract surgery, where the we to "be very accurate in the calculation of the intraocular lense." On the other hand, nowadays we also have new devises oriented to facilitate the diagnosis thanks to the technological advances of the last years, both referring to design engineering, modeling, and construction as the use of new methodologies that allow the analysis and treatment of the data. For example, new image modalities, improvements in the visualization systems, algorithms that allow automatic and objective extraction of relevant data, systems that develop the Big- Data to extract knowledge, etc... have appeared. Examples of this are the corneal topographers that allow us to explore and study the eyeball in a very accurate way, the OCT systems (Optical Coherence Tomography) dedicated to study and analyse the back of the eye, or, the most recent system the angio-OCT, that allows us to perform tests to study retina vascular diseases without the use of dyes, being a noninvasive test. Other developments and researches that sound like fiction occur in the field of artificial vision: "bionic eyes" are being worked on or artificial vision with chips for the retina, which on patients that have an important visual loss can distinguish lumps and shapes or big letters. In the next year's spectacular contributions and developments are expected. The developments, researches, and clinical studies are important, and are what allow the progress to occur; that said, as important as this is to have the tools that allow its disclosure to society and to the scientific community, serving in turn as the starting point in the pursuit and approaches to new challenges. Eye Care and Vision (ECV) is set up with that purpose, being a completely open publication whose objective is the disclosure of any progress in the vision care field, always based on one principle: to be meticulous in the quality of its contributions. We hope that the impact of this publication reaches legal age in the coming years and becomes a referent in the "vision care" field.
Copyright: ( 2017 Penedo MG. This is an open-access article distributed under the terms of the Creative Commons Attribution License, which permits unrestricted use, distribution, and reproduction in any medium, provided the original author and source are credited.
Correspondence to: Manuel G. Penedo, University of Coruña, Spain, E-mail: mgpenedo@udc.es

Received: May 03, 2017; Accepted: May 15, 2017; Published: May 18, 2017 